\title{
Potential Synergism of Caffeic Acid Phenethyl Ester and Dasatinib in C6 Glioma Cell Model: Adumbrating the Molecular Mechanism
}

\author{
Henah Mehraj Balkhi ${ }^{1 *}$, Taseen Gul' ${ }^{1}$ Syed Sana ${ }^{2}$ and Ehtishamul Haq ${ }^{1}$ \\ ${ }^{1}$ Department of Biotechnology, University of Kashmir, Jammu and Kashmir, India \\ ${ }^{2}$ Centre for Research and Development, University of Kashmir, Jammu and Kashmir, India
}

\begin{abstract}
Background: Gliomas are one of the most invasive, highly recurrent, heterogeneous cancers resistant to most of the current treatment regimes and hence almost incurable. CAPE and Dasatinib when used in a congruous combination and durations, present an antitumor potential for glioma.

Objective: CAPE and Dasatinib in combination have been shown to inhibit proliferation and induce apoptosis in C6 glioma cells. However, the signaling pathway of their antiproliferative and apoptotic effects remains unknown. In this study, the antiproliferative effects of combination treatment on C6 glioma cells were investigated.

Methods: Expression analysis of proteins thought to be mediating proliferation, cell motility, angiogenesis, and invasion was carried out to delineate the molecular mechanism entailing antineoplastic action of CAPE and Dasatinib.

Results: Co-treatment induces a change in cellular and nuclear morphology followed by apoptosis and a significant decrease in the activity of catalase and MMP-2, Pro-MMP 2, MMP-9 and Pro-MMP 9 in C6 glioma cells. Moreover, CAPE and Dasatinib modulate the expression of proteins having potential interactive crosstalk with major oncogenic pathways involved in glioma progression. Our results showed that combination treatment modulates the expression of p53, ERK1/2, and AKT in C6 glioma cells. p53, EGFR and PCNA transcript expressions were attuned in co-treated C6 cells.

Conclusion: Importantly, antineoplastic effects of CAPE and Dasatinib were far greater than those afforded by treatment with a single drug. Together these drugs reduce glioma proliferation and invasion felicitously implying that combination treatment could be a useful therapy for treatment of glioma.
\end{abstract}

Keywords: Caffetic acid phenethyl ester; Dasatinib; Durg therapy; Glioma; Synergistic effect

\section{Introduction}

Gliomas are one of the most aggressive forms of cancer [1]. Accounting for $80 \%$ of malignant CNS (central nervous system) tumors they are one of the most common primary brain tumors [2,3]. Gliomas have mutated, aberrantly expressed transmembrane receptors and their downstream effectors in ways that constitutively activates/ deactivates different pathways [1]. Gliomas are one of the most invasive cancers infiltrating into surrounding brain matter since they are highly limited by the skull they rarely metastasize outside the central nervous system. The treatment of gliomas represents a significant challenge to oncologists [2]. Failure to treat with an agent that targets one molecule may result from the uninhibited action of other multiple crosstalks or from the need to target more than one oncogenic signaling cascade concurrently.

Dasatinib is an ATP-competitive small molecule protein kinase inhibitor, approved clinically for the treatment of chronic myeloid leukemia [3]. Dasatinib exhibits a broad spectrum of protein kinase inhibition including BCR-ABL, Src, c-kit, platelet-derived growth factor receptor and ephA2 $[4,5]$. Src an oncoprotein is a non-receptor tyrosine kinase family member that has pleiotropic functions within the cell. Src functions in a complex with various signaling molecules and modulate cytoskeletal organization, cell motility, invasion, and cell division [6]. Src can phosphorylate EGFR and PDGFR, leading to increased mitogen-activated protein kinase (MAPK) and phosphatidylinositol 3-kinase pathway signaling thus promoting cellular transformation and mitogenesis [6]. Thus, SFKs play a pivotal role in signaling downstream from various receptor tyrosine kinases and other signaling pathways that are critical both for normal cell and tumor cell proliferation, invasion, and survival[7]. Dasatinib has been reported to inhibit the growth and migration of solid tumors in vitro through Src inhibition. In vitro and in vivo studies support a potentially important role of Src in gliomas [8]. Preclinical data suggest that Dasatinib has a broader spectrum of molecular targets and increased potency against key targets than other receptor tyrosine kinase inhibitors [9-12].

Caffeic acid phenethyl ester (CAPE) is the active component derived from propolis purified from the hives of honeybees [12,13]. CAPE has biological activities like anti-proliferation and anti-inflammation, immunomodulatory and neuroprotective properties [9-12]. In vitro and in vivo studies substantiate the neuroprotective potential of CAPE [13-17]. It has been illustrated that CAPE is a potent and specific inhibitor of NF- $\kappa B$ activation [18]. CAPE is able to inhibit ischemiainduced neuronal death and degeneration of neurons [19]. CAPE treatment also inhibits NO-induced neurotoxicity and protects isolated mitochondria against cytochrome $\mathrm{C}$ release induced by calcium ions.

Natural extracts target multiple pathways, thus lowering the druginduced resistance in glioma. Targeting cell signaling cascades involved

*Corresponding author: Henah Mehraj Balkhi, Department of Biotechnology, University of Kashmir, Jammu and Kashmir, India 190006, Tel: 9596572354; E-mail:henahbalkhi@gmail.com

Received March 08, 2018; Accepted April 12, 2018; Published April 18, 2018

Citation: Balkhi HM, Gul T, Sana S, Haq E (2018) Potential Synergism of Caffeic Acid Phenethyl Ester and Dasatinib in C6 Glioma Cell Model: Adumbrating the Molecular Mechanism. Brain Disord Ther 7: 245. doi: 10.4172/2168-975X.1000245

Copyright: (c) 2018 Balkhi HM, et al. This is an open-access article distributed under the terms of the Creative Commons Attribution License, which permits unrestricted use, distribution, and reproduction in any medium, provided the original author and source are credited. 
in glioma with a single agent have yielded disappointing results. Combinations of drugs that inhibit multiple cell signaling cascades represent an attractive therapeutic strategy to achieve a better outcome. Previous studies have shown treatment with CAPE and Dasatinib inhibited cell proliferation, induced cell death and prolonged changes in glial cell morphology in C6 glioma cells [20]. In the present study, we addressed the question whether CAPE and Dasatinib target multiple processes involved in glioma proliferation, invasion, and development. Expression analysis of most pivotal proteins of some crucial pathways was carried out to delineate the molecular mechanism entailing antineoplastic action of CAPE and Dasatinib. Thus we evaluated the effect of CAPE and Dasatinib on the expression of proteins thought to be mediating proliferation, cell motility, angiogenesis, and invasion. Here we report CAPE and Dasatinib co-treatment targets MMPs (matrix metalloproteinase); proteins known to be involved in the process of cellular invasion, p53 (cellular tumor antigen p53) and ERK1/2 (extracellular signal-regulated kinases), involved in many cellular processes. The apoptotic and antiproliferative activity of combination therapy could be further attributed to its ability to target AKT (protein kinase B). This pathway is highly regulated by multiple mechanisms, often involving cross-talk with other signaling pathways. Transcription of EGFR (epidermal growth factor receptor) which lies at the junction of many intracellular signaling pathways was also found to be downregulated. PCNA (proliferating cell nuclear antigen) a proliferative marker in gliomas regulating one of the crucial processes of cell cycle entry and progression was downregulated on treatment with combination therapy. Interpreting to mean CAPE and Dasatinib seem to target pathways most intricately involved in the processes of gliomagenesis i.e. p14ARF/MDM2/p53, Ras-Raf-MEK-ERK and RTK/ $\mathrm{PI} 3 \mathrm{~K} / \mathrm{AKT} / \mathrm{mTOR}$ axes [21].

\section{Methods}

\section{Materials}

PI (propidium iodide) and Fura-2 AM (Fura-2 pentakis (acetoxymethyl) ester) were bought from Sigma-Aldrich, Co. St Louis, MO, USA, 100\% HPLC purified CAPE was purchased from Calbiochem, Dasatinib was purchased from Guangzhou Eastbang Pharmaceutical Technology Co., China. Sybrgreen mix was brought from Thermo Scientific Inc., protein quantification assay kit from BioRad Laboratories, Inc., PVDF (polyvinylidene difluoride) membrane was brought from Millipore-USA. All other chemicals were of ultrapure grade and obtained from Sigma-Aldrich, Co. St Louis, MO, USA, or otherwise indicated.

\section{Cell culture and treatments}

C6 cell line derived from Rattus Norvegicus was used as in vitro model of glioma. The cells were cultured, sub cultured and cryopreserved as per instructions (ATCC/ CCL-107). C6 cells were incubated to $85-95 \%$ confluency and for all the experiments working cell density used was $2 \times 10^{4} \mathrm{cells} / \mathrm{ml}$ or otherwise indicated. CAPE was dissolved in Dimethyl sulfoxide while as Dasatinib was dissolved in Phosphate-buffered saline.

\section{Quantification of apoptosis by propidium iodide staining}

PI, a membrane impermeant and apoptotic indicator stain was used to quantify apoptosis in C6 cells. After treatment, the culture medium was aspirated and each well was gently washed with cold PBS (Phosphate-buffered saline) for 5 min twice. After washing, cells were incubated with $5 \mu \mathrm{g} / \mathrm{ml}$ PI solution for $30 \mathrm{~min}$ in the dark. The cells were then washed with Tris buffer (50 mM Tris-HCl, $\mathrm{pH}$ 7.3). Wells containing only PI were taken as a control to determine the level of the background signal. Wells containing cells and treated with $0.2 \%$ Triton $\mathrm{X}-100$ (10-15 min treatment) were taken to determine the maximum fluorescence signal from dead cells. The samples were kept at $4^{\circ} \mathrm{C}$ throughout the washing and staining procedure to minimize changes in the living to dead cell distributions as a consequence of manipulation and staining. Stained cells were monitored by the Floid cell imaging station (Life Technologies, California, USA). After exposure to the condition, six different fields of view were chosen randomly for observations. PI fluorescence intensities were measured using Image J software and presented as fold increase versus non-treated control.

\section{Detection of calcium levels by Fura-2 AM fluorescent assay}

The cytoplasmic levels of calcium were examined using Fura-2 AM a fluorescent calcium indicator. At the end of the experiment, cells were washed once with PBS and once with fura- $2 \mathrm{AM}$ buffer $(137 \mathrm{mM} \mathrm{NaCl}$, $2.7 \mathrm{mM} \mathrm{KCl}, 1 \mathrm{mM} \mathrm{Na} \mathrm{HPO}_{3}, 25 \mathrm{mM}$ glucose, $20 \mathrm{mM}$ Hepes). Cells were then incubated at $37^{\circ} \mathrm{C}$ in the above buffer with $5 \mu \mathrm{M}$ fura- $2 \mathrm{AM}$ for $30 \mathrm{~min}$ in the dark. After incubation, the cells were washed three times with the same buffer solution but without fura- $2 \mathrm{AM}$ at $37^{\circ} \mathrm{C}$. To determine the maximum and minimum calcium signal, $0.1 \%$ Triton $\mathrm{X}-100$ and $4.5 \mathrm{mM}$ ethylene glycol tetraacetic acid were added along with fura buffer respectively. Fluorescence emission was captured using the Floid Cell Imaging Station (Life Technologies, California, USA). Fluorescence changes within the cells were converted to reflect the changes in calcium levels. Fluorescence response was measured in six randomly chosen fields of view in three separate experiments after exposure to the condition.

\section{Protein extraction and quantitation}

After the required experimentation, culture media was removed from cell culture dishes and washed with pre-chilled PBS twice and scraped into lysis buffer (20 mM TrisCl (pH 8), $137 \mathrm{mM} \mathrm{NaCl}, 10 \%$ Glycerol, 1\% Nonidet P-40, and $2 \mathrm{mM}$ EDTA, $1 \mathrm{mM}$ PMSF, $5 \mathrm{mM}$ $\mathrm{NaF}$ and phosphatase inhibitors). Cells were incubated at $4^{\circ} \mathrm{C}$ for 60 min. After the treatment samples were centrifuged at $12000 \mathrm{rpm}$ for $10 \mathrm{~min}$. The supernatants were transferred to freshly chilled Eppendorf tubes and protein samples were frozen at $-80^{\circ} \mathrm{C}$ until further use. For enzymatic assays; extracellular culture media and all samples were

\begin{tabular}{|c|c|c|c|c|}
\hline \multicolumn{5}{|c|}{ Primer pair p53 (Product length 150) } \\
\hline Direction & Sequence $\left(5^{\prime}->3^{\prime}\right)$ & Length & $\mathrm{Tm}$ & GC\% \\
\hline Forward primer & CAGAGGAAGCCCTCCAAGTG & 20 & $66.2^{\circ} \mathrm{C}$ & 60.00 \\
\hline Reverse primer & r GGTGGAAGCCATAGTTGCCT & 20 & $65.2^{\circ} \mathrm{C}$ & 55.00 \\
\hline \multicolumn{5}{|c|}{ Primer pair PCNA (Product length 160) } \\
\hline Direction & Sequence $\left(5^{\prime}->3^{\prime}\right)$ & Length & $\mathrm{Tm}$ & GC\% \\
\hline Forward primer & AACTCCGCCACCATGTTTGA & 20 & $67.7^{\circ} \mathrm{C}$ & 50.00 \\
\hline Reverse primer & GCTGCACTAAGGAGACGTGA & 20 & $63.2^{\circ} \mathrm{C}$ & 55.00 \\
\hline \multicolumn{5}{|c|}{ Primer pair EGFR (Product length 119) } \\
\hline Direction & Sequence $\left(5^{\prime}->3^{\prime}\right)$ & Length & Tm & GC\% \\
\hline $\begin{array}{l}\text { Forward } \\
\text { primer }\end{array}$ & GATCACGGCTCGTGTGTCC & 19 & $67.1^{\circ} \mathrm{C}$ & 63.16 \\
\hline $\begin{array}{l}\text { Reverse } \\
\text { primer }\end{array}$ & ATGCCTATGCCATTGCAAACT & 21 & $65.3^{\circ} \mathrm{C}$ & 42.86 \\
\hline \multicolumn{5}{|c|}{ Primer Pair $\beta$-Actin (Product Length 101) } \\
\hline Direction & Sequence $\left(5^{\prime}->3^{\prime}\right)$ & Length & $\mathrm{Tm}$ & GC\% \\
\hline $\begin{array}{l}\text { Forward } \\
\text { primer }\end{array}$ & TTCCTTCCTGGGCATGGAGT & 20 & 60.85 & 55.00 \\
\hline $\begin{array}{l}\text { Reverse } \\
\text { primer }\end{array}$ & CTGTGTTGGCGTACAGGTCT & 20 & 59.97 & 55.00 \\
\hline
\end{tabular}

Table 1: Sequence and characteristic properties of gene-specific forward and reverse primers for respective gene amplification. 
prepared adequately to maintain the function of the enzymes and used immediately after collection. A Bio-Rad Laboratories protein assay kit based on Bradford's method was used for measuring protein concentration spectrophotometrically at $595 \mathrm{~nm}$ as per instructions in the manual.

\section{Catalase in-gel assay}

In order to evaluate the effect of drug treatment on catalase activity, an in-gel assay was performed. Samples containing equal amounts of protein $(50 \mu \mathrm{g})$ were separated on native gradient PAGE. When the achromatic zones in the gel started to appear, the stain was poured off and the gel was gently washed with double distilled water. Zymograms were scanned with a Bio-Rad GS-800 calibrated densitometer (Hercules, CA, USA). Each band was scanned three times and the area showing enzymatic activity was calculated using software supplied by Bio-Rad.

\section{Matrix metalloproteinase activity by gelatin zymography}

Effect of drug treatment on MMP-2 and MMP-9 expression in C6 cells was examined. Samples adjusted to equal protein concentration and volume were mixed thoroughly with $2 \mathrm{X}$ Laemmli sample buffer [0.125 M Tris-HCl, $\mathrm{pH} 6.8,4 \%$ (w/v) SDS (sodium dodecyl sulfate), $20 \%(\mathrm{v} / \mathrm{v})$ glycerol, $0.04 \%(\mathrm{w} / \mathrm{v})$ bromophenol blue], and subjected to gelatinase zymography on $10 \%$ SDS-acrylamide gel with $0.1 \%$ gelatin under non-reducing conditions. Following electrophoresis, the gels were washed twice in $2.5 \%$ Triton X-100 for 30 min at room temperature to remove SDS and four times in water before overnight incubation at $37^{\circ} \mathrm{C}$ in substrate buffer $\left(50 \mathrm{mM}\right.$ Tris- $\mathrm{HCl}, 10 \mathrm{mM} \mathrm{CaCl}_{2}$, $1 \mu \mathrm{M} \mathrm{ZnCl}_{2}$, and $0.01 \%$ sodium azide) and stained with $0.5 \%$ Coomassie Blue R-250 in 50\% methanol and 10\% glacial acetic acid for $30 \mathrm{~min}$ and destained. Gelatinase zymograms were scanned and gelatinolytic bands were measured densitometrically with a Bio-Rad GS-800 calibrated densitometer (Hercules, CA, USA). Each band was scanned three times and the area was calculated using software supplied by Bio-Rad.

\section{Western blotting analysis}

Protein extract was preheated at $100^{\circ} \mathrm{C}$ for $5 \mathrm{~min}$ in reducing SDS sample buffer (50 mM Tris- $\mathrm{HCl}(\mathrm{pH} 6.8), 2 \%$ SDS, $10 \%$ glycerol, $0.1 \%$ bromophenol blue and $100 \mathrm{mM} \beta$-mercaptoethanol). Samples were adjusted to equal protein concentration and volume and subjected to SDS-PAGE (sodium dodecyl sulfate-polyacrylamide gel electrophoresis) on 10-12\% SDS-acrylamide gel with a discontinuous buffer system. Separated proteins were transferred to PVDF membrane by a semi-dry transfer method in accordance with the manufacturer's instructions (Hoefer-USA) for 1 hour at $200 \mathrm{~mA}$. For immunedetection, the PVDF membrane was processed and blocked with 5\% bovine serum albumin in PBS for three hours at room temperature. After blocking membranes were washed twice with PBST $(0.2 \%$ Tween-20) and then incubated with the respective specific primary antibodies; p53, AKT (1:1000; Santa Cruz Biotechnologies Dallas Taxas, US) and ERK (1:1000; Cell Signaling) Anti $\beta$-actin, anti $\alpha$-tubulin (1:7000; Sigma-Aldrich) were used as loading control. After overnight incubation at $4^{\circ} \mathrm{C}$ temperature, membranes were washed thrice for 10 minutes with PBST (0.5\% Tween). The secondary detection was performed using anti-mouse IgG Dylight 680 conjugate $(1: 10,000)$ or anti-rabbit IgG Dylight 800 conjugate $(1: 10,000)$ secondary antibodies. After washing three times for 10 minutes with PBST, Odyssey infrared detection system (LI-COR, Lincoln, NE, U.S.A.) was used to detect Western blots and band intensity. The results were represented as expression levels of specific gene normalized to $\beta$-actin and $\alpha$-tubulin relative to the normalized expression of the gene in control cells.

\section{RNA extraction and real-time quantitative PCR}

Total RNA from samples was extracted using Trizol reagent (Invitrogen, USA) in accordance with the manufacturer's instructions. The quantity and integrity of total RNA were determined by electrophoresing the total isolated RNA through $1.0 \%$ denaturing agarose gel stained with ethidium bromide by analyzing gel on Image Quant LAS 4000 (GE Healthcare Life Sciences, Buckinghamshire, UK) (Supplementary Data 1; Figures S1-S4). The RNA concentrations were measured at $290 \mathrm{~nm}$ with Nanodrop 2000 UV-Visible Spectrophotometer (Thermo Scientific, Massachusetts, USA). The first strand $\mathrm{cDNA}$ was generated from $5 \mu \mathrm{g}$ of total RNA in a final volume of $20 \mu \mathrm{l}$ using Moloney Murine Leukemia Virus reverse transcriptase (Thermo Scientific) and random hexamers. PCR amplification was performed using Benchmark DNA thermal cycler (TC 9639). cDNA synthesis was performed as follows: $25.0^{\circ} \mathrm{C}, 7 \mathrm{~min} ; 40.0^{\circ} \mathrm{C}, 60 \mathrm{~min}$; $65^{\circ} \mathrm{C}, 15 \mathrm{~min}$. To further analyze the specificity of primers and quality of cDNA to be used for qRT-PCR (quantitative Real-Time-PCR), endpoint PCR of the obtained cDNA was carried out with specific primers and Taq DNA polymerase. To determine the expression level of each gene qRT-PCR was done with specific primer pairs for each gene and $\beta$-actin as a loading control. The $3.5 \mu \mathrm{l}$ of synthesized cDNA was amplified in a total volume of $25 \mu \mathrm{l}$ in Real Time PCR 7500 (Applied Systems) using maxima sybrgreen mix and in accordance with manufacturer's protocol. qRT-PCRs were performed in a MicroAmp optical 96-well plate (Applied Biosystems). All the reactions were run in triplicates as follows: $94^{\circ} \mathrm{C}, 30 \mathrm{~s} ; 60^{\circ} \mathrm{C}, 30 \mathrm{~s} ; 72^{\circ} \mathrm{C}, 90 \mathrm{~s} ; 40$ cycles, finally extending at $72^{\circ} \mathrm{C}$ for $5 \mathrm{~min}$. For primer designing the referential genomic DNA sequence was derived from GenBank, a gene sequence database at the National Center for Biotechnology Information (NCBI;http://www.ncbi.nlm.nih.gov) and Ensemble (http://www. ensembl.org). The sequences for gene-specific forward and reverse primers were designed using NCBI-Primer Blast software (http://www. ncbi.nlm.nih.gov/primer-blast). The details of the primers used in this study are shown in Table 1. Ct (Cycle threshold), was determined for each sample, and the average $\mathrm{Ct}$ of triplicate samples was calculated. For characterization of the generated amplicons and to control for contamination by unspecific by-products, a melting curve analysis was applied between $60^{\circ} \mathrm{C}-95^{\circ} \mathrm{C}$ with the Real-time PCR 7500 (Applied Systems) using the fluorescent dye sybrgreen (Supplementary Data 2) (Figure S5-S7). In addition, the PCR product size was verified by electrophoresis on $2 \%$ agarose gel containing $0.1 \mathrm{mg} / \mathrm{ml}$ of ethidium bromide comparing their mobilities with those of molecular weight marker (Supplementary Data 2) (Figure S2-S4).

\section{Statistical analysis}

All the experiments were repeated at least three times and results are expressed as Mean \pm SD of three experiments carried out individually. All the data was analyzed using One Way ANOVA by Dunnett's multiple comparisons tests using GraphPad Prism 5.

\section{Results}

\section{Preliminary results}

In order to evaluate the effect of CAPE and Dasatinib on proliferation of C6 cells we initially carried out MTT assay. C6 cells were treated with CAPE over a concentration range of $10 \mu \mathrm{M}$ to $110 \mu \mathrm{M}$ and incubated for $24 \mathrm{~h}$ along with untreated cell control and medium control wells to provide the blanks for absorbance readings. In parallel, cells were treated with Dasatinib over a concentration range of $0.12 \mathrm{nM}$ to $0.875 \mathrm{nM}$. The proliferation of C6 cells decreased on treatment with CAPE and Dasatinib in concentration dependent manner consistently, compared to control. After $24 \mathrm{~h}$ of incubation, before density limitation 
Citation: Balkhi HM, Gul T, Sana S, Haq E (2018) Potential Synergism of Caffeic Acid Phenethyl Ester and Dasatinib in C6 Glioma Cell Model: Adumbrating the Molecular Mechanism. Brain Disord Ther 7: 245. doi: 10.4172/2168-975X.1000245

of cell growth occurred in control wells MTT assay was performed. A significant proliferation decrement of C6 cells treated with increasing concentrations of CAPE and Dasatinib was detected. There was about $50 \%$ reduction in cell proliferation at $60 \mu \mathrm{M}$ of CAPE and at $0.5 \mathrm{nM}$ Dasatinib (results not shown). Concentrations at which inhibition in cell proliferation was $25 \%$ (IC-25) was $40 \mu \mathrm{M}$ and $0.2 \mathrm{nM}$ respectively. Therefore, for further studies with the combination of CAPE and Dasatinib we choose the most optional concentrations of CAPE and Dasatinib, IC-25 concentrations; the semi inhibitory doses.

\section{CAPE and Dasatinib co-treatment induces cell damage accompanied by apoptosis}

We carried out PI staining which is an index of cell damage and apoptosis under nonfixed cell staining conditions thus delineating morphological changes induced by stress from changes in morphology due to apoptosis. Results showed that the treatment of C6 cells with CAPE and Dasatinib in combination significantly induced cell apoptosis in comparison to control group (Figure 1A). In the control group, nuclei stained with PI were only $\sim 1 \%$, in CAPE treated group $\sim 4 \%$ and Dasatinib treatment induced apoptosis in C6 cells by $\sim 13.3 \%$,

A
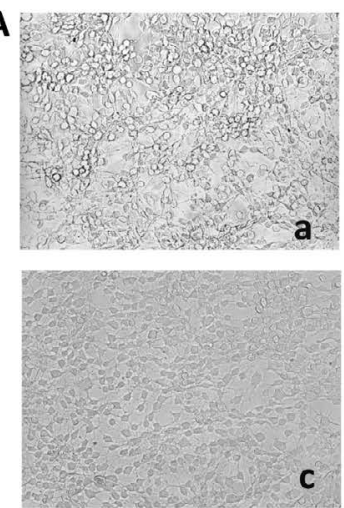

C
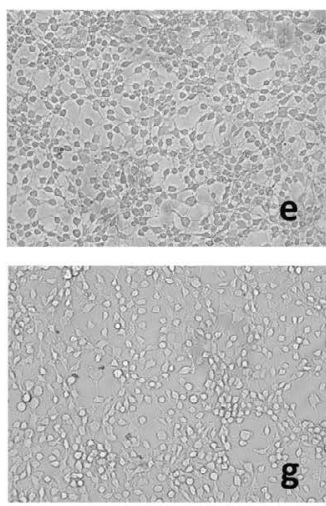

g
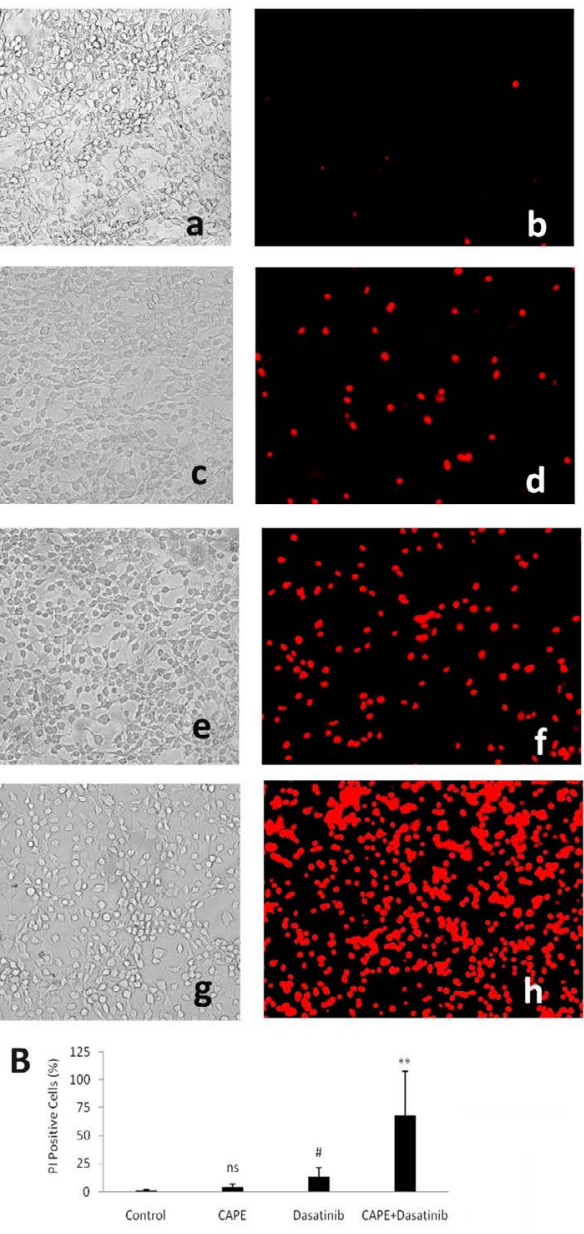

Figure 1: (A) Representative micrographs showing, BW images (Lane 1) and Pl-stained corresponding fluorescent images (Lane 2) (scale bar: $100 \mu \mathrm{m}$ ). Nuclei stained with PI were fewer in control $(a, b)$ in comparison to CAPE; $40 \mu \mathrm{M}$ (c, d) or Dasatinib; $0.2 \mathrm{nM}(\mathrm{e}, \mathrm{f})$ treated group, CAPE+ Dasatinib $(\mathrm{g}, \mathrm{h})$ treated cells showed maximum marked granular apoptotic bodies. (B) Fluorescence intensities measured using image $\mathrm{J}$ software are presented as means \pm SDs $(n=3){ }^{* *} p<0.0001, \# p<0.001$.
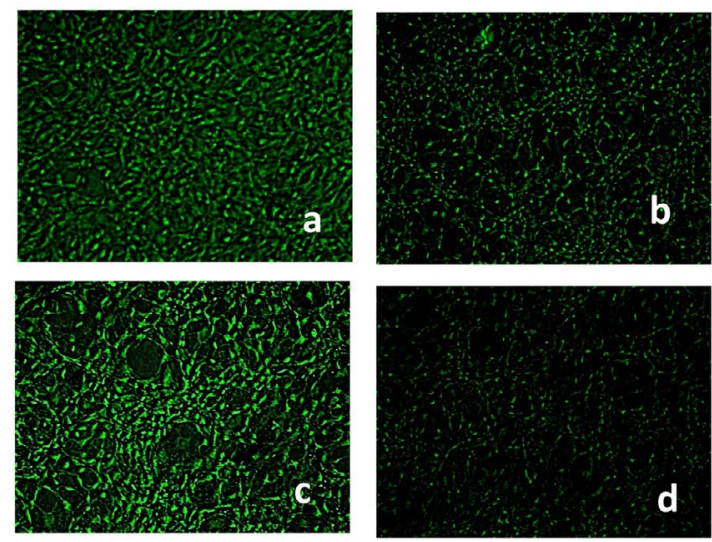

Figure 2: Representative fluorescent photomicrographs showing calciuminduced fluorescence after staining with fura-2AM (scale bar: $100 \mu \mathrm{M}$ ). Control (a) cells showed maximum fluorescence which gradually decreased in cells treated with CAPE; $40 \mu \mathrm{M}$ (b) or Dasatinib; $0.2 \mathrm{nM}$ (c), the maximum reduction in fluorescence was observed in CAPE+ Dasatinib (d) group indicating attenuation of intracellular calcium levels in C6 glioma cell culture.

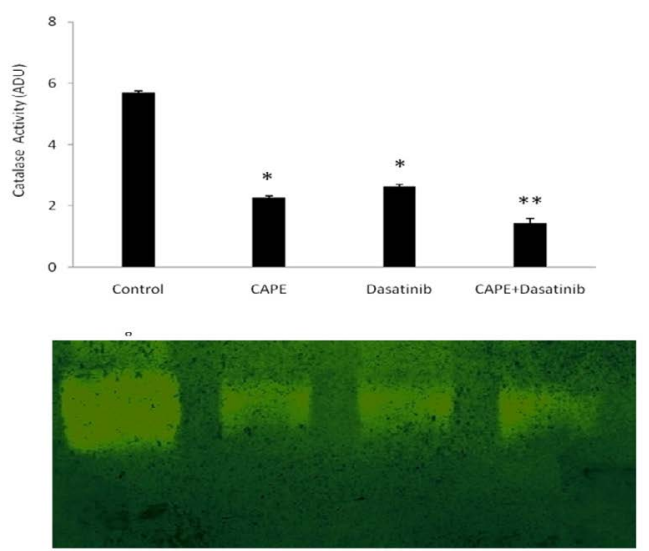

Figure 3: Catalase activity in an 'in-gel' catalase activity assay in CAPE $(40 \mu \mathrm{M})$, Dasatinib $(0.2 \mathrm{nM})$ and combination treated groups. Catalase activity decreased in CAPE and Dasatinib group; however decrease in activity was predominant in CAPE + Dasatinib group, activity levels are presented as means \pm SDs $(n=3)$ ** $p<0.0001,{ }^{*} p=0.0001$ in ADU (arbitrary densitometric units).

while as $\sim 68 \%$ of the co-treated cells were PI stained (Figure 1B). According to this characterization, we concluded that co-treatment leads to morphological changes induced by apoptosis in C6 glioma cells.

\section{Fura-2 AM staining: Corroborative to inhibitory effect on} intracellular calcium

An approach of labeling with fura-2 AM was taken to ascertain the inhibitory effect of drug treatment on intracellular calcium levels. As shown in Figure 2, staining with fura-2 AM indicated high calciuminduced fluorescence in C6 cells without treatment. Fluorescence gradually decreased in cells treated with CAPE or Dasatinib, indicating CAPE and Dasatinib attenuate intracellular calcium levels in C6 glioma cell culture. CAPE and Dasatinib co-treatment showed relatively lower levels of calcium in terms of lesser fluorescence intensity.

\section{Downregulation in expression of catalase protein: Affirmation} from in-gel assay

In order to determine the effect of drug treatment on antioxidant defense system in C6 glioma cells, catalase expression was studied by 
activity gel. The obtained results indicated a decrease in expression of catalase in CAPE and Dasatinib treated group while as a co-treated group showed a predominant decrease in expression (Figure 3). As shown by densitometric analysis, catalase activity decreased by $\sim 3.5$ units in CAPE treated group, $\sim 3$ units in Dasatinib treated group and $\sim 4.5$ units decrease was observed in the co-treated group.

\section{Effect on intracellular and extracellular MMP-2 and MMP-9 activity}

C6 cells invade the extracellular matrix with the help of the activity of metalloproteases. To investigate whether CAPE and Dasatinib treatment have an effect on expression of MMP-2 and MMP-9 activity gelatin zymography assay was carried out with cell lysates as well as with the respective culture media samples separately. Treatment of C6 cells with CAPE and Dasatinib in combination for $24 \mathrm{~h}$ resulted in a significant decrease in MMP-2, Pro-MMP 2, MMP-9 and Pro-MMP 9 activity in comparison to control, CAPE and Dasatinib only group in cell lysates (Figure 4). MMP-2 activity was reduced by $\sim 1.3$ units in CAPE treated; 23 units in Dasatinib treated and 3.5 units in the co-treated group. Pro-MMP 2 activity was reduced by $\sim 0.5$ unit in CAPE treated, $\sim 2$ units in Dasatinib treated and $\sim 2.4$ unit in the cotreated group (Figure 4A). Densitometric quantification showed a $\sim 2$ unit decrease in MMP-9 activity in CAPE and Dasatinib treated group and $\sim 3$ unit decrease in the co-treated group. Similarly, Pro-MMP 9 activity was reduced by $\sim 0.6$ units in CAPE treated, $\sim 1.5$ in Dasatinib treated and $\sim 2$ units in the co-treated group (Figure $4 \mathrm{~B}$ ). Next, we probed culture media of the above samples for expression analysis of extracellular MMP-2, Pro-MMP 2, MMP-9 and Pro-MMP 9. A significant decrease in MMP-2 and Pro-MMP 2 activity was induced by drug treatment (Figure 5A). Densitometric analysis showed a decrease
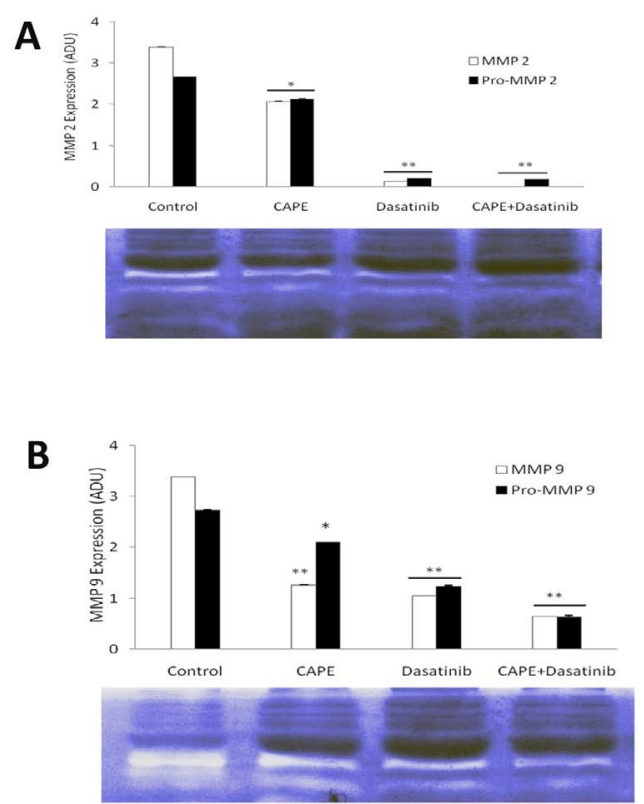

Figure 4: Zymogram showing expression of MMP- 2, Pro-MMP 2, MMP-9 and Pro-MMP 9 in whole cell lysates after treatment with CAPE $(40 \mu \mathrm{M}))$, Dasatinib $(0.2 \mathrm{nM})$ and in combination. (A) CAPE and Dasatinib in combination for 24 $\mathrm{h}$ resulted in a significant decrease in MMP- 2 and Pro-MMP 2 activity in comparison to control, CAPE and Dasatinib only group. Expression levels are presented as means \pm SDs $(n=3) * p<0.0001$ in ADU (arbitrary densitometric units) (B) CAPE and Dasatinib in combination for $24 \mathrm{~h}$ resulted in a significant decrease in MMP- 9 and Pro-MMP 9 activity in comparison to control, CAPE and Dasatinib only group. Expression levels are presented as means \pm SDs $(n=3){ }^{* *} p<0.0001,{ }^{*} p=0.0001$ in ADU.
A

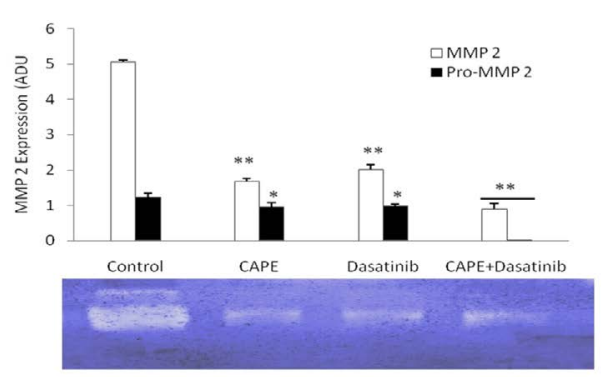

B

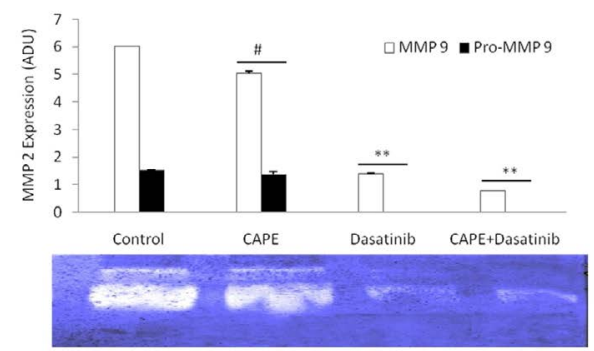

Figure 5: Zymograms showing expression of MMP-9 Pro-MMP 9, MMP-2 and Pro-MMP 2 in extracellular media after treatment with CAPE $(40 \mu \mathrm{M}))$, Dasatinib (0.2 nM) and in combination. (A) CAPE and Dasatinib in combination for $24 \mathrm{~h}$ resulted in a significant decrease in MMP-2 and Pro-MMP 2 activity in comparison to control, CAPE and Dasatinib only group. Expression levels are presented as means \pm SDs $(n=3){ }^{* *} p<0.0001$, $\# p<0.001$ in ADU (arbitrary densitometric units) (B) Zymogram showing decrease in MMP-9, Pro-MMP 9, activity in co-treated group in comparison to control, CAPE and Dasatinib only group. Expression levels are presented as means \pm SDs $(n=3){ }^{* *} p<0.0001$, $\# p<0.001$ in ADU.

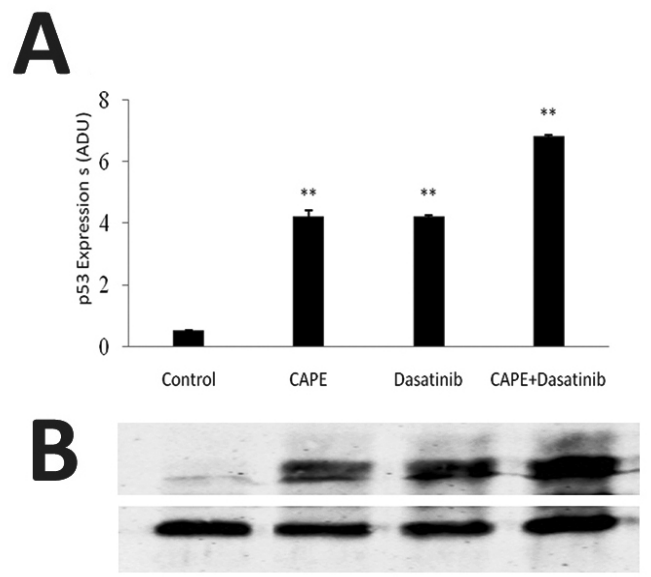

Figure 6: (A) Immunoblots showing the expression of p53 protein in C6 glioma cells after treatment with CAPE, Dasatinib and in combination. The p53 protein is upregulated in the co-treated group when compared with control, CAPE and Dasatinib only groups, $\beta$-actin (Lower Panel) was used as loading control and expression in each group was normalized to $\beta$-actin relative to the normalized expression in control cells. (B) Expression levels are presented as means \pm SDs $(n=3){ }^{* *} p<0.0001$ in ADU (arbitrary densitometric units).

in activity of MMP-2 by $\sim 3.3$ unit in CAPE, $\sim 3$ units in Dasatinib and $\sim 4$ units in the co-treated group. There was no significant reduction in Pro-MMP 2 in CAPE, and Dasatinib only group, however, there was $\sim 1.2$ unit decrease in activity in the co-treated group. Further densitometric quantification showed MMP-9 activity was reduced by $\sim 1$ unit in CAPE, $\sim 4.6$ unit in Dasatinib and $\sim 5.3$ unit in the co-treated group. Correspondingly Pro-MMP 9 activity was reduced by $\sim 0.2$ units 
in CAPE, 1.4 units in Dasatinib and $~ 1.5$ units in the co-treated group (Figure 5B). Besides it was observed that expression of active forms of MMP-2 and MMP-9 was higher in cell culture media in comparison to cell lysates of same treatment group. However, expression of Pro MMP-9 and Pro MMP-2 was higher in cell lysates in comparison to cell culture media of same treatment group.

\section{CAPE and Dasatinib induces p53 expression in C6 glioma} cells

p53 is one of the major tumor suppressor proteins in gliomas. Glioma tumors retain wild-type p53; hence ability to maintain the tumor phenotype depends on downregulated expression of p53. Since CAPE and Dasatinib induce apoptosis, to ascertain whether p53 is involved in drug-induced apoptosis, Western blotting was employed. Our results showed that CAPE and Dasatinib co-treatment

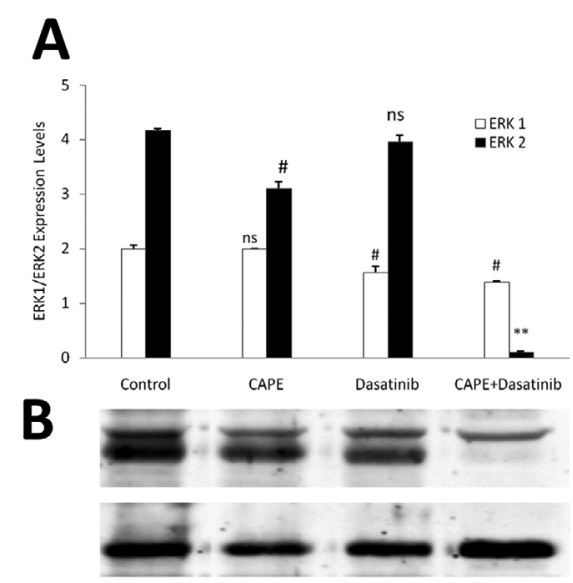

Figure 7: Immunoblots showing the expression of the ERK1/2 protein in C6 glioma cells after treatment with CAPE, Dasatinib and in combination. ERK1/2 protein is downregulated in the co-treated group when compared with control, CAPE and Dasatinib only groups, -tubulin (Lower Panel) was used as loading control and expression in each group was normalized to a-tubulin relative to the normalized expression in control cells. Expression levels are presented as means \pm SDs $(n=3){ }^{* *} p<0.0001, \# p<0.001$ in ADU (arbitrary densitometric units).

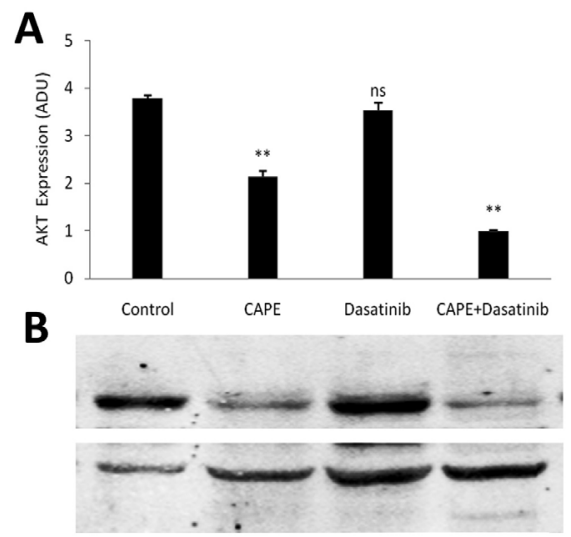

Figure 8: Immunoblots showing the expression of AKT protein in C6 glioma cells after treatment with CAPE, Dasatinib and in combination. AKT protein is downregulated in the co-treated group when compared with control, CAPE and Dasatinib only groups, $\beta$-actin (Lower Panel) was used as loading control and expression in each group was normalized to $\beta$-actin relative to the normalized expression in control cells. Expression levels are presented as means \pm SDs $(n=3){ }^{* *} p<0.0001$ in ADU (arbitrary densitometric units). induced p53 expression robustly in C6 glioma cells compared with the control, CAPE and Dasatinib only group (Figure 6A). Furthermore, quantification of the protein blots after normalization with $\beta$-actin expression indicated that $\mathrm{p} 53$ expression was $\sim 4$ unit higher in samples treated with CAPE and Dasatinib while as there was $\sim 6$ unit increase in $\mathrm{p} 53$ expression in group co-treated with CAPE and Dasatinib compared to the control samples (Figure 6B). Thus, the combination of CAPE and Dasatinib significantly induces expression of p53, impeding growth and proliferation of C6 glioma cells.

\section{Co-treatment with CAPE and Dasatinib significantly decreases the expression of ERK1/2 in C6 glioma cells}

We have demonstrated that co-treatment with CAPE and Dasatinib decreased the cell proliferation and migration in C6 cells. So, we further investigated the main proliferation signaling regulatory pathway in glioma, the Ras/Raf/MAPK pathway. MAPK (also termed as ERK $1 / 2$ or $\mathrm{p} 42 / \mathrm{p} 44$ ) is the terminal enzyme in this signaling cascade, so ERK1/2 appeared to be an important regulatory molecule in the whole cascade to be evaluated. As shown in Figure 7A, the expression of ERK appreciably decreased in the co-treated group as compared to the control, CAPE and Dasatinib only group. $\alpha$ - Tubulin levels were detected in the protein extract to demons trate equal loading and for normalization of protein expression (Figure 7B). Furthermore, quantification of the protein blots indicated that ERK1 expression was $\sim 0.7$ units less in co-treated samples as compared to the control samples and $\sim 0.5$ unit decrease was observed in Dasatinib treated group, however, there was no significant reduction in expression of ERK1 in CAPE treated group. ERK2 expression was downregulated by $\sim 1$ unit in CAPE treated group, there was no significant change in expression of ERK2 in Dasatinib treated group. However co-treated group showed a highly significant downregulation in ERK2 expression by $\sim 4$ units. The decrease in the expression of ERK1/2 in the co-treated group further confirms the antineoplastic activity of this combination therapy.

\section{In vitro effects of CAPE and Dasatinib on the AKT pathway}

RTK/PTEN/PI3K pathway is a signal transduction pathway that promotes survival and growth in response to extracellular signals in gliomas. AKT represents a nodal point in this pathway which allows for uncontrolled proliferation, evasion of apoptosis, and enhanced tumor invasion. So the next question we asked was if the CAPE and Dasatinib co-treatment would inhibit expression of AKT or not. The effect of CAPE and Dasatinib treatment on AKT pathway was evaluated by Western blot analysis. CAPE treatment markedly inhibited AKT expression while as Dasatinib alone had a less modest effect on AKT expression while as AKT expression was significantly down regulated in the co-treatment group (Figures $8 \mathrm{~A}$ and $8 \mathrm{~B}$ ). Densitometric analysis of immunoblots after normalization with $\beta$-actin expression depicts a $\sim 1.6$ unit decrease in expression of AKT in CAPE treated group, there was only an insignificant change of $\sim 0.3$ units in Dasatinib group. However co-treated group showed a highly significant reduction in expression of AKT by $\sim 2.8$ units.

\section{CAPE and Dasatinib co-treatment upregulates p53 mRNA expression to a significant extent}

p53 is regulated very intricately at transcriptional, translational, as well as intermolecular levels. Considering this complexity, after analysis of protein expression we further studied the expression of $\mathrm{p} 53$ by qRTPCR. To continue our analysis, total RNA was isolated from cell lysates after drug treatment, qRT-PCR was performed to quantify p53 in the samples. Our results showed that the expression of p53 was $\sim 6$ fold 
up-regulated in the samples treated with the combination of CAPE and Dasatinib as compared to the control samples. The upregulation of p53 mRNA was also detected in samples treated with CAPE and Dasatinib only, but the increase in expression was only $~ 1.6$ fold in CAPE treated group and 2.7 fold in Dasatinib group (Figure 9). The values represent p53 expression levels normalized to $\beta$-actin relative to the normalized expression of p53 gene in control cells. These results were consistent with those obtained by western blotting indicating that treatment with CAPE and Dasatinib regulates p53 expression at all levels.

\section{EGFR expression is suppressed by CAPE and Dasatinib co- treatment in $\mathrm{C} 6$ glioma cells}

EGFR genes have been reported as one of the most important mediators for malignant progression of gliomas. Hence we implored the effect of drug treatment on EGFR expression in C6 glioma cells. In the present study, qRT-PCR was used to examine the EGFR inhibition effects of drug treatment on C6 glioma cells. Interestingly, qRT-PCR of the total RNA extracted from C6 cells, using EGFR specific primers showed a decrease in the expression of EGFR in the co-treated group as compared to untreated C6 cells. Our results demonstrated that CAPE and Dasatinib co-treatment inhibits EGFR expression, exerting growth inhibition effect on C6 glioma cells in vitro. In comparison to control EGFR expression was decreased by $\sim 2$ fold in CAPE treated group and $\sim 1.3$ fold in Dasatinib treated group while as EGFR expression was most significantly reduced by $\sim 2.6$ fold in the co-treated group (Figure 10). The values represent EGFR expression levels normalized to $\beta$-actin relative to the normalized expression of EGFR gene in control cells. Consistent with our previous results co-treatment significantly minimizes C6 cell proliferation and malignant progression.

\section{PCNA down-regulation in C6 cells induced by CAPE and Dasatinib co-treatment}

PCNA is a nuclear protein, a cofactor for DNA polymerase,

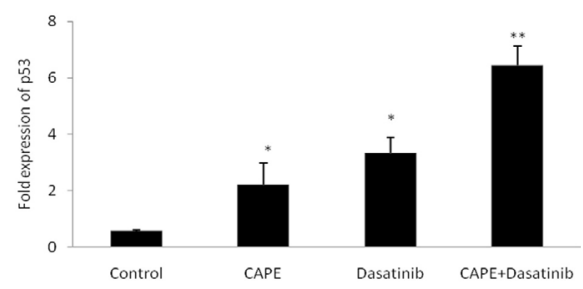

Figure 9: qRT-PCR for assessing the expression of p53 in C6 glioma cells with CAPE, Dasatinib, and co-treatment. There was a significant upregulation of $p 53$ in co-treated samples when compared with control, CAPE and Dasatinib group. Values expressed are means \pm SDs $(n=3)$ of fold expression of p53 in different groups relative to control normalized to $\beta$-actin, ${ }^{* *} p<0.0001,{ }^{*} p=0.0001$ between indicated groups.

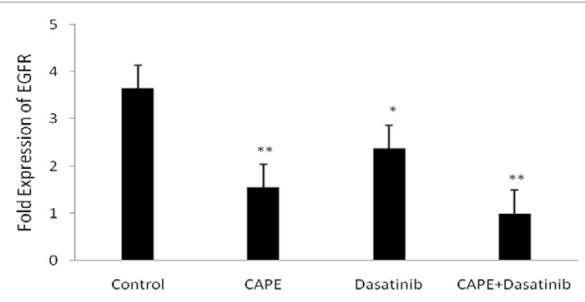

Figure 10: qRT-PCR for assessing the expression of EGFR in C6 glioma cells with CAPE, Dasatinib, and co-treatment. EGFR was found to be downregulated in co-treated samples when compared with control, CAPE and Dasatinib group. Values expressed are means \pm SDs $(n=3)$ of fold expression of EGFR in different groups relative to control normalized to $\beta$-actin, ** $p<0.0001,{ }^{*} p=0.0001$ between indicated groups.

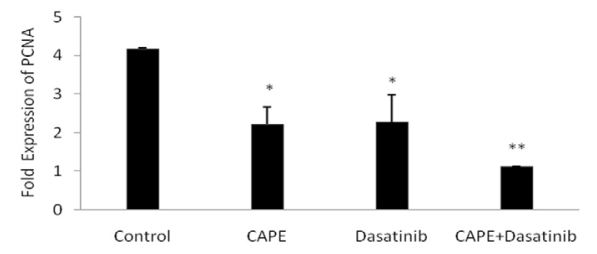

Figure 11: qRT-PCR for assessing the expression of PCNA in C6 glioma cells with CAPE, Dasatinib, and co-treatment. PCNA was found to be significantly downregulated in co-treated samples when compared with control, CAPE and Dasatinib group. Values expressed are means \pm SDs $(n=3)$ of fold expression of PCNA in different groups relative to control normalized to $\beta$-actin, ${ }^{* *} p<0.0001$, ${ }^{*} p=0.0001$ between indicated groups.

the major enzyme for the replication in eukaryotes. PCNA is well recognized as a proliferative marker in gliomas. So next, we asked whether drug treatment regulates transcription of PCNA or not. PCNA transcript levels were assessed by qRT-PCR. Our results showed that the expression was highest in the control cells and decreased in the groups treated with CAPE and Dasatinib by $\sim 2$ folds. A significant $\sim 3$ fold decrease in expression was detected in the group treated with both CAPE and Dasatinib (Figure 11). The values represent PCNA expression levels normalized to $\beta$-actin relative to the normalized expression of PCNA gene in control cells.

\section{Discussion}

Gliomagenesis is a multifactorial process involving an intricate interaction between a number of signaling cascades resulting in unrestricted proliferation and invasion $[22,23]$. The invasive and proliferative nature of glioblastoma has been one of the impetuses for testing of new therapies to more expeditiously mitigate tumorigenic invasion, proliferation, and survival. Since glioma is a molecularly complex disease thus treatment failure with a single agent that targets one signaling abnormality may result from the uninhibited actions of other molecular abnormalities or from the need to target more than one oncogenic signaling cascade simultaneously. Therefore, treatment with agents that could target several key signaling pathways represents an attractive therapeutic approach.

Dasatinib is an orally bioavailable inhibitor of tyrosine kinases like SRC, KIT, PDGFR, EPHA2, and BCR-ABL fusion. Dasatinib is effective in impeditive activity in multiple malignancies. It is also an approved drug for BCR-ABL mutant hematological malignancies. Therefore, we hypothesized that Dasatinib might be more effective for gliomas than other receptor tyrosine kinase inhibitors. However, studies have revealed that Dasatinib alone did not provide many benefits in mouse models of human glioblastoma. Because some tumors and cell types have shown to be refractory to Dasatinib monotherapy, so we argued that a combination therapy with Dasatinib may be a viable clinical option for resistant tumors.

Compounds from natural extracts with effective biological activities are being researched for various anti-cancer activities. Natural extracts target multiple pathways in diseases which could lower drug-induced resistance in tumors, by targeting numerous signaling processes thus inhibiting the proliferation and growth of glioma cells. The propolis of honeybee hives has been used in folk medicine since ages as antiinflammatory agents with a certain anticancer activity. CAPE one of the active components of propolis has a variety of in vitro pharmacological activities. It is known to have anti-mitogenic, anticarcinogenic activities in various malignancies and suppresses acute immune and inflammatory responses. 
Citation: Balkhi HM, Gul T, Sana S, Haq E (2018) Potential Synergism of Caffeic Acid Phenethyl Ester and Dasatinib in C6 Glioma Cell Model: Adumbrating the Molecular Mechanism. Brain Disord Ther 7: 245. doi: 10.4172/2168-975X.1000245

This study was carried out to assess the efficacy of Dasatinib and CAPE co-treatment against gliomas. CAPE and Dasatinib co-treatment inhibited proliferation and invasiveness in C6 cells otherwise resistant to the treatment of same drugs at same concentrations singly [20]. Cotreatment with CAPE and Dasatinib not only induces apoptosis in C6 cells but also inhibits cellular clonogenicity as well, indicating CAPE and Dasatinib target different cellular processes in glioma. Our results showed that CAPE and Dasatinib initiate the process of apoptosis in C6 glioma cells. Calcium ions are involved in a number of cellular signaling cascades in the brain during tumorigenesis which influences a multitude of cellular reactions due to the diversity of calcium-binding proteins. Calcium ions also play a critical role in glutamate excitotoxicity in brain [24-32]. Co-treatment decreases the calcium levels in C6 glioma cells; imperatively it would influence the whole process of gliomagenesis via calcium signaling. Catalase a primary antioxidant enzyme plays a very important role in the defense against oxidative stress in the brain as such it is constitutively elevated in glioma cells when compared to their normal cell counterpart [33]. Upon cotreatment with CAPE and Dasatinib, the activity of catalase significantly decreased thus countering the stress resistance mechanism in C6 cells [27,28]. Gliomagenesis is a multifactorial process that involves a complex interplay between extracellular matrix, adjacent cells and glial cells which result in active proliferation and invasion of tumor cells into the adjacent boundaries ${ }^{23}$. Cellular proliferation and invasiveness is a highly regulated process and the loss of control on these processes stems from a loss of inhibitory regulation [29]. One of the most understood mechanisms of C6 cell invasion is by metalloprotease activity. MMPs cause proteolytic processing of extracellular matrix structural proteins, thus regulating cell migration and invasion [30]. Co-treatment with CAPE and Dasatinib resulted in a significant decrease in MMP-2 and MMP-9 activity as well as in the expression of active forms of MMP-2 and MMP-9. Glioma, as indicated by recent studies, seems to results from sequential inactivation of the p14ARF/ MDM2/p53, RTK/PI3K/Akt/mTOR axes and Ras/MEK/MAPK pathway [21]. $70-80 \%$ of gliomas analyzed harbor alterations in all three pathways [31]. In particular, we studied the effect of CAPE and Dasatinib on the expression of proteins having potential interactive cross-talk with other oncogenic pathways during glioma progression and interconnecting these signaling pathways and betwixt them. p53 is one of the major tumor suppressor proteins in gliomas. However, glioma tumors retain wild-type p53; hence the ability to maintain the tumor phenotype depends on downregulated expression of $\mathrm{p} 53$. Since co-treatment with CAPE and Dasatinib induces apoptosis so we investigated the expression of p53 in C6 cells with and without treatment. Co-treatment with CAPE and Dasatinib induced p53 expression in C6 glioma cells compared with the control, CAPE and Dasatinib only group. Thus CAPE and Dasatinib co-treatment induced reactivation of p53 impeding tumor growth and proliferation of C6 glioma cells. Up-regulation of p53 is believed to be instrumental in cell growth inhibition. However, it has been found that in some cancer cell lines the p53 genomic changes did not overtly correlate to the expression of $\mathrm{p} 53$ protein $[32,33]$. It has been indicated that the elevation of 553 protein levels in response to DNA damage occurs in the absence of clear changes in mRNA levels [34]. Sometimes moderate increases in mRNA expression are followed by large increases in protein levels [35]. Considering this complexity, after expression analysis of protein we further studied the expression of p53 by qRTPCR. At transcriptional levels, CAPE and Dasatinib co-treatment induced p53 expression in C6 glioma cells compared with the control, CAPE and Dasatinib only group. Thus, a combination of CAPE and Dasatinib induces expression of p53 not only at protein level only but at the transcriptional level also. In gliomas, in addition to p53 pathway, the MAPK (mitogen-activated protein kinase) is also known to be activated. MAPKs are serine-threonine protein kinases with a multitude of downstream effects. These molecules transduce various stimuli from cell surface leading to activation of transcriptional factors through sequential protein phosphorylations. The activity of RTK (receptor tyrosine kinase) plays an important role in cell fate decision, cell proliferation and migration during neural development and adult neurogenesis [36,37]. Signaling of RTKs results in activation of the Ras-Raf-MEK-ERK pathway in gliomas. ERK1/2 a member of MAPK signaling pathway results in persistent activation of the ERK-MAPK cascade through multiple pathways [38]. ERKs are known to play a major role in various cellular responses. ERK pathway is involved in cell growth, proliferation, differentiation, migration in cell systems. Different pools of ERK in various subcellular locations are activated by different isoforms of Protein kinase $\mathrm{C}$ (PKC) resulting in different glial phenotypes and varied regulation of cellular processes. Similarly, it has been observed that increase in intracellular cAMP strongly inhibits ERK activity in C6 cells and enhances the rate of differentiation into astrocytic phenotype indicating a possible role for ERK in the negative regulation of GFAP expression and glioma cell differentiation. Rhoassociated protein kinase (ROCK), a serine-threonine kinase has also been reported to be involved mainly in regulating the shape, migration, and movement of cells by acting on the cytoskeleton via linkage to the ERK signaling pathway [39]. Depletion of ROCK1/2 decreased the expression of cyclin D1 and PCNA. Inhibitor of ERK was able to downregulate the expression of cyclin D1 and PCNA showing this effect is regulated through ERK [40-43]. The oncogenic potential of ERK was demonstrated in part by the finding that ERK-1/2 activity was elevated in untreated C6 cells while as the expression of ERK appreciably decreased in the co-treated cells. Downregulation of ERK expression is an important predictor of antineoplastic activity of combination treatment. AKT is a downstream serine/threonine kinase in the RTK/ PTEN/PI3K pathway. The pathway is involved in cell survival, inhibiting apoptosis through phosphorylation and inactivation of various target molecules; in addition, it also plays a critical role in metabolism. Studies have revealed that this pathway is mutated in the majority of gliomas and AKT plays a central role in this signal transduction pathway. This pathway is highly regulated by multiple mechanisms, often involving cross-talk with other signaling pathways. Enough studies have shown that AKT can be activated in response to increases in cellular calcium levels, via Calcium/Calmodulin dependent protein kinase. We have earlier showed CAPE and Dasatinib cotreatment modulates calcium levels in C6 cells, thus a highly significant reduction in expression of $\mathrm{AKT}$, as well as modulation in calcium levels, implies that CAPE and Dasatinib target not the only expression of nodal proteins but also the secondary molecules. Studies have proven that AKT contributes to angiogenesis by activating endothelial nitric oxide synthase, which increases production of nitric oxide and our previous studies have shown that CAPE and Dasatinib co-treatment decreased nitric oxide levels in C6 cells [20]. Based on these observations we can overtly conclude that the CAPE and Dasatinib target not only the multiple pathways but also betwixt them and the intracellular molecules as well. EGFRs are overexpressed in gliomas and play a significant role in regulating other intracellular signaling pathways. Since EGFR is involved in both mTOR/PI3K/AKT and RAS/MAPK pathway, therefore, we evaluated the effect of CAPE and Dasatinib cotreatment on transcription of EGFR. From the results, we could clearly understand that reduction in EGFR expression in the co-treated group further implies that this therapy targets multiple pathways. PCNA is well recognized as a proliferative marker in gliomas. Studies have 
Citation: Balkhi HM, Gul T, Sana S, Haq E (2018) Potential Synergism of Caffeic Acid Phenethyl Ester and Dasatinib in C6 Glioma Cell Model: Adumbrating the Molecular Mechanism. Brain Disord Ther 7: 245. doi: 10.4172/2168-975X.1000245

Page 9 of 10

revealed that PCNA plays a coordinating role and has a striking ability to interact with multiple partners who are involved in critical cellular activities including [41] DNA synthesis and repair, cell cycle regulation and apoptosis. Myriad of cytosolic proteins contain PCNA interacting motif (APIM) and the PCNA-interacting peptide (PIP)-box and several of these proteins are involved in signaling cascades such as MAPK and $\mathrm{PI} 3 \mathrm{~K} / \mathrm{Akt}^{41}$. ERK8 also contains APIM and the interaction is found to stabilize PCNA. Targeting high levels of cytosolic PCNA with APIMpeptide induces caspase activation leading to apoptosis in multiple myeloma cells. Similarly in neuroblastoma cells cytosolic PCNA was shown to bind and stabilize procaspase-9 [41]. PCNA transcript levels were assessed after drug treatment since it is a nuclear protein and cofactor for DNA polymerase so it's downregulated expression would suppress cell cycle entry and progression. The mechanism by which such potentiation could be achieved would involve the inhibition of cell proliferation, invasion, and the inhibition of angiogenesis.

Glioma is complex and multidimensional in nature; with diseases having interrelationships with different organs as well as with other parts of the brain as such there are additional complications in evaluating the effects of drug therapy compared to other malignancies. As this was an in vitro study, replication of in vivo environment was challenging. It is not possible to simulate the metabolic consecution of drugs in vivo from in vitro experiments as the brain has a very complex microenvironment, vast cell types and cellular interactions and organization which are not possible to mimic under in vitro conditions. The doses and duration of drug treatment cannot be extrapolated from in vitro concentrations. Further, it is difficult to surmise the consequences of long-term drug exposures and all relevant mechanisms of toxicity in normal cell subsets from this study. In addition to the above-noted limitations regarding the in vivo environment, there is an additional limitation of biasing of the treatment outcome based on disease severity and staging in vivo which could not be accounted under in vitro conditions. Besides the practical considerations for patients when they're putting the agents together, relevant to dosing and administration schedules cannot be accounted from in vitro study. Clearly, more work is needed to be carried out in vivo glioma models at least to mimic microenvironment in brain tumor patients. Adequately powered, high-quality descriptive studies are needed.

\section{Conclusion}

Since there is a substantial need for a chemotherapy that is more effective as well as safe, this in vitro study was necessary to establish combining agents to try to more effectively suppress the aberrant signaling pathways by using rationally designed combinations of therapeutics. Despite these limitations, relevant conclusions from this study have helped to Figure out how to use a rational combination of drugs that have a strong complementary antitumor effect that is verified in a relevant in vitro model. This study was an effort to put agents together that has a limited overlap of their toxicities and has limited detrimental potential pharmacologic interactions. In conclusion, we have described multi-targeting effects of CAPE and Dasatinib cotreatment on glioma cells. Co-treatment inhibits multiple cellular processes involved in tumorigenesis hence decreasing the possibility of drug-induced resistances. These drugs are not only addictive but highly synergistic in activity. CAPE dramatically decreased the dose of Dasatinib needed to achieve inhibition of glioma cell proliferation suggesting that perhaps using two agents together increases the therapeutic potential of drugs thus can be an effective therapy for treating glioma.

\section{References}

1. Wen PY, Kesari SN (2008) Malignant gliomas in adults. Engl J Med 359: 492507.

2. Siegel R, Ma J, Zou Z, Jemal A (2014) Cancer statistics. C.A. Cancer J Clin 64: $9-29$

3. Nakada M, Kita D, Watanabe T, Hayashi Y, Teng L, et al. (2011) Aberrant signaling pathways in glioma. Cancers 3: 3242-78.

4. Maher EA, Furnari FB, Bachoo RM, Rowitch DH, Louis DN, et al. (2001) Malignant glioma: genetics and biology of a grave matter. Genes Development 15: $1311-1333$.

5. Lombardo LJ, Lee FY, Chen P, Norris D, Barrish JC, et al. (2004) Discovery of $\mathrm{N}$-(2-chloro-6-methyl- phenyl)-2-(6-(4-(2-hydroxyethyl)- piperazin-1-yl)-2methylpyrimidin-4- ylamino)thiazole-5-carboxamide (BMS-354825), a dual Src/ Abl kinase inhibitor with potent antitumor activity in preclinical assays. J Med Chem 47: 6658-6661.

6. Doggrell SA (2005) BMS-354825: a novel drug with potential for the treatment of imatinib-resistant chronic myeloid leukaemia. Expert Opin Investig Drugs 14: 89-91

7. Hiwase DK, Saunders V, Hewett D, Frede A, Zrim S, et al. (2008) Dasatinib cellular uptake and efflux in chronic myeloid leukemia cells: therapeutic implications. Clinical cancer research : an official journal of the American Association for Cancer Research 14: 3881-8.

8. Roskoski $R$ (2004) Src protein-tyrosine kinase structure and regulation Biochem Biophys Res Commun 324: 1155-1164.

9. Hayakawa F, Naoe T (2006) SFK-STAT pathway: an alternative and important way to malignancies. Ann N Y Acad Sci 1086: 213-222.

10. Ahluwalia MS, de Groot J, Liu WM, Gladson CL (2010) Targeting SRC in glioblastoma tumors and brain metastases: rationale and preclinical studies. Cancer lett 298: 139-149.

11. Grunberger D, Banerjee R, Eisinger K, Oltz EM, Efros L, et al. (1988) Preferential cytotoxicity on tumor cells by caffeic acid phenethyl ester isolated from propolis. Experientia 44: 230-232.

12. Su ZZ, Grunberger D, Fisher PB (1991) Suppression of adenovirus type 5 E1A-mediated transformation and expression of the transformed phenotype by caffeic acid phenethyl ester (CAPE). Mol Carcinog 4: 231-242.

13. Lin J, Su Z, Grunberger D, Zimmer S, Fisher P (1994) Expression of the transformed phenotype induced by diverse acting viral oncogenes mediates sensitivity to growth suppression induced by caffeic Acid phenethyl ester (cape). Int J Oncol 5: 5-15.

14. Su ZZ, Lin J, Grunberger D, Fisher PB (1994) Growth suppression and toxicity induced by caffeic acid phenethyl ester (CAPE) in type 5 adenovirustransformed rat embryo cells correlate directly with transformation progression. Cancer Res 54: 1865-1870.

15. Hwang HJ , Park HJ , Chung HJ, Min HY, Park EJ, et al. (2006) Inhibitory effects of caffeic acid phenethyl ester on cancer cell metastasis mediated by the down-regulation of matrix metalloproteinase expression in human HT1080 fibrosarcoma cells. J Nutri Biochem 17: 356-362.

16. Onori P, DeMorrow S, Gaudio E, Franchitto A, Mancinelli R, et al. (2009) Caffeic acid phenethyl ester decreases cholangiocarcinoma growth by inhibition of NFkappaB and induction of apoptosis. Int J Cancer 125: 565-576.

17. Lin WL, Liang WH, Lee YJ, Chuang SK, Tseng TH (2010) Antitumor progression potential of caffeic acid phenethyl ester involving p75(NTR) in C6 glioma cells. Chem Biol Interact 188: 607-615.

18. Wang LC, Chu KH, Liang YC, Lin YL, Chiang BL (2010) Caffeic acid phenethy ester inhibits nuclear factor-kappaB and protein kinase $B$ signalling pathways and induces caspase- 3 expression in primary human CD4+ T cells. Clin Exp Immunol160: 223-232.

19. Fontanilla CV, Wei X, Zhao L, Johnstone B, Pascuzzi RM, et al. (2012) Caffeic acid phenethyl ester extends survival of a mouse model of amyotrophic latera sclerosis. Neuroscience 205: 185-193.

20. Natarajan K, Singh S, Burke TR, Grunberger D, Aggarwal BB (1996) Caffeic acid phenethyl ester is a potent and specific inhibitor of activation of nuclear transcription factor NF-kappa B. Proc Natl Acad Sci U S A 93: 9090-9095.

21. Amodio R, De Ruvo C, Sacchetti A (2003) Caffeic acid phenethyl ester blocks 
Citation: Balkhi HM, Gul T, Sana S, Haq E (2018) Potential Synergism of Caffeic Acid Phenethyl Ester and Dasatinib in C6 Glioma Cell Model: Adumbrating the Molecular Mechanism. Brain Disord Ther 7: 245. doi: 10.4172/2168-975X.1000245

Page 10 of 10

apoptosis induced by low potassium in cerebellar granule cells. Int $\mathrm{J}$ Dev Neurosci 21: 379-389.

22. Balkhi HM, Gul T, Haq E (2016) Anti-neoplastic and calcium modulatory action of caffeic acid phenethyl ester and dasatinib in C6 glial cells: a therapeutic perspective. CNS Neurol Disord Drug Targets15: 54-63.

23. Sunayama J, Matsuda KI, Sato A, Tachibana K, Suzuki K, et al. (2010) Crosstalk between the $\mathrm{PI} 3 \mathrm{~K} / \mathrm{mTOR}$ and MEK/ERK pathways involved in the maintenance of self-renewal and tumorigenicity of glioblastoma stem-like cells. Stem cells 28: 1930-1939.

24. Inoue S, Ichikawa T, Kurozumi K (2012) Novel animal glioma models that separately exhibit two different invasive and angiogenic phenotypes of human glioblastomas. World Neurosurg 78: 670-682.

25. Onishi M, Ichikawa T, Kurozumi K, Date I (2011) Angiogenesis and invasion in glioma. Brain Tumor Pathol 28: 13-24.

26. Balkhi HM GT, Banday MZ, Haq E (2014) Glutamate excitotoxicity: An Insight into the Mechanism. Int J Adc Res 2: 361-373.

27. Bernstein M, Lyons SA, Moller T, Kettenmann H (1996) Receptor-mediated calcium signalling in glial cells from mouse corpus callosum slices. J Neurosci Res 46: 152-163.

28. Lau A, Tymianski M (2010) Glutamate receptors, neurotoxicity and neurodegeneration. Pflugers Archiv 460: 525-542.

29. Smith PS, Zhao W, Spitz DR, Robbins ME (2007) Inhibiting catalase activity sensitizes 36B10 rat glioma cells to oxidative stress. Free Radic Biol Med 42: 787-797

30. Zhong W, Yan T, Lim R, Oberley LW (1999) Expression of superoxide dismutases, catalase, and glutathione peroxidase in glioma cells. Free Radic Biol Med 27: 1334-1345.

31. Demuth T, Berens ME (2004) Molecular mechanisms of glioma cell migration and invasion. J Neurooncol 70: 217-228.

32. Lakka SS, Jasti SL, Gondi C, Boyd D, Chandrasekar N, et al. (2002)
Downregulation of MMP-9 in ERK-mutated stable transfectants inhibits glioma invasion in vitro. Oncogene 21: 5601-5608.

33. Sherr CJ, McCormick F (2002) The RB and p53 pathways in cancer. Cancer Cell 2: 103-112.

34. Hsu HC, Tseng HJ, Lai PL, Lee PH, Peng SY (1993) Expression of p53 gene in 184 unifocal hepatocellular carcinomas: association with tumor growth and invasiveness. Cancer Res 53: 4691-4694.

35. Hsu IC, Tokiwa T, Bennett W, Metcalf RA, Welsh JA, et al. (1993) p53 gene mutation and integrated hepatitis $B$ viral DNA sequences in human liver cancer cell lines. Carcinogenesis 14: 987-992.

36. Kubbutat MH, Vousden $\mathrm{KH}$ (1998) Keeping an old friend under control: regulation of p53 stability. Mol Med Today 4: 250-256.

37. Maltzman W, Czyzyk L (1984) UV irradiation stimulates levels of p53 cellula tumor antigen in nontransformed mouse cells. Mol Cell Biol 4: 1689-1694.

38. Mandell JW, Hussaini IM, Zecevic M, Weber MJ, VandenBerg SR (1998) In situ visualization of intratumor growth factor signaling: immunohistochemical localization of activated ERK/MAP kinase in glial neoplasms. Am J Pathol 153 1411-23.

39. Kapoor GS, O'Rourke DM (2003) Mitogenic signaling cascades in glial tumors Neurosurgery. 52: 1425-34; discussion 34-5

40. Xia Z, Dickens M, Raingeaud J, Davis RJ, Greenberg ME (1995) Opposing effects of ERK and JNK-p38 MAP kinases on apoptosis. Science 270: 1326 1331

41. Zohrabian VM, Forzani B, Chau Z, Murali R , Jhanwar-Uniyal M (2009) Rho/ROCK and MAPK signaling pathways are involved in glioblastoma cell migration and proliferation. Anticancer Res 29: 119-123.

42. Zhao Y, Lv M, Lin H, Cui $Y$, Wei XQ, et al. (2013) Rho-associated protein kinase isoforms stimulate proliferation of vascular smooth muscle cells through ERK and induction of cyclin D1 and PCNA. Biochem Biophys Res Commun 432: 488-493.

43. Maga G , Hubscher U (2003) Proliferating cell nuclear antigen (PCNA): a dancer with many partners. J Cell Sci 116: 3051-3060. 